\title{
Spatial scales of marine conservation management for breeding seabirds
}

4 Steffen Oppel $^{1, *}$, Mark Bolton ${ }^{1}$, Ana P. B. Carneiro ${ }^{2}$, Maria P. Dias ${ }^{2}$, Jonathan A. Green ${ }^{3}$, Juan F. Masello ${ }^{4}$, Richard A. Phillips ${ }^{18}$, Ellie Owen ${ }^{1}$, Petra Quillfeldt ${ }^{4}$, Annalea Beard ${ }^{5}$,

6 Sophie Bertrand ${ }^{6,7}$, Jez Blackburn ${ }^{8}$, P. Dee Boersma ${ }^{9}$, Alder Borges ${ }^{10}$, Annette C.

Broderick $^{11}$, Paulo Catry ${ }^{12}$, Ian Cleasby ${ }^{1}$, Elizabeth Clingham ${ }^{5}$, Jeroen Creuwels ${ }^{13}$, Sarah

8 Crofts $^{14}$, Richard J. Cuthbert ${ }^{1}$, Hanneke Dallmeijer ${ }^{15}$, Delia Davies ${ }^{16}$, Rachel Davies ${ }^{17}$, Ben Dilley $^{16}$, Herculano Andrade Dinis ${ }^{21}$, Justine Dossa ${ }^{2}$, Michael J Dunn ${ }^{18}$, Marcio A. Efe ${ }^{19}$,

Annette L. Fayet ${ }^{20}$, Leila Figueiredo ${ }^{19}$, Adelcides Pereira Frederico ${ }^{10}$, Carina Gjerdrum ${ }^{22}$, Brendan J. Godley ${ }^{11}$, José Pedro Granadeiro ${ }^{24}$, Tim Guilford ${ }^{20}$, Keith C Hamer ${ }^{25}$, Carolina Hazin $^{2}$, April Hedd ${ }^{26}$, Leeann Henry ${ }^{5}$, Marcos Hernández-Montero ${ }^{27}$, Jefferson Hinke ${ }^{28}$, Nobuo Kokubun $^{29}$, Eliza Leat ${ }^{30}$, Benjamin Metzger ${ }^{31}$, Teresa Militão ${ }^{23}$, Gilson Montrond ${ }^{10}$, Wim Mullié ${ }^{15}$, Oliver Padget ${ }^{20}$, Elizabeth Pearmain ${ }^{2}$, Ingrid L. Pollet ${ }^{32}$, Klemens Puetz ${ }^{33}$, Flavio Quintana ${ }^{34}$, Norman Ratcliffe ${ }^{18}$, Robert A. Ronconi ${ }^{22}$, Peter G. Ryan ${ }^{16}$, Sarah Saldanha ${ }^{35}$, Akiko Shoji ${ }^{20}$, Jolene $\mathrm{Sim}^{30}$, Cleo Small ${ }^{1}$, Louise Soanes ${ }^{3}$, Akinori Takahashi ${ }^{29}$, Phil Trathan ${ }^{18}$, Wayne Trivelpiece ${ }^{28}$, Jan Veen ${ }^{15}$, Ewan Wakefield ${ }^{36}$, Nicola Weber ${ }^{30,11}$, Sam Weber $^{30,11}$, Laura Zango ${ }^{23}$, Francis Daunt, Motohiro Ito, Michael P. Harris, Mark A. Newell, Sarah Wanless, Jacob González-Solís ${ }^{23}$, John Croxall ${ }^{2}$

${ }^{1}$ RSPB Centre for Conservation Science, Royal Society for the Protection of Birds, United Kingdom

${ }^{2}$ BirdLife International, David Attenborough Building, Cambridge CB2 3QZ, United Kingdom

${ }^{3}$ School of Environmental Sciences, University of Liverpool, Liverpool, L69 3GP, United Kingdom

${ }^{4}$ Department of Animal Ecology \& Systematics, Justus Liebig University Giessen, HeinrichBuff-Ring 26, D-35392 Giessen, Germany

${ }^{5}$ Marine Section, Environmental and Natural Resources Directorate, St Helena Government, St Helena Island, South Atlantic Ocean.

${ }^{6}$ IRD, UMR Marbec - Centre de Recherche Halieutique, Avenue Jean Monnet, 34200 Sète

${ }^{7}$ Universidad Federal Rural de Pernambuco, Rua Dom Manuel de Medeiros, s/n, Dois Irmãos

- CEP: 52171-900 - Recife/PE, Brazil

${ }^{8}$ British Trust for Ornithology, The Nunnery, Thetford, Norfolk, IP24 2PU, UK.

${ }^{9}$ Center for Ecosystem Sentinels, University of Washington, 24 Kincaid Hall, Seattle, WA 98195-1800, USA

${ }^{10}$ Projecto Vitó, Achada de São Filipe, São Filipe, Ilha do Fogo, Cabo Verde

${ }^{11}$ Centre for Ecology and Conservation, University of Exeter, Cornwall Campus TR10 9EZ, UK 
${ }^{12}$ MARE - Marine and Environmental Sciences Center, ISPA - Instituto Universitário.

Portugal.

${ }^{13}$ Naturalis Biodiversity Center, P.O. Box 9517, 2300 RA Leiden, The Netherlands

${ }^{14}$ Falklands Conservation, Stanley, Falkland Islands FIQQ 1ZZ

${ }^{15}$ Veda Consultancy, Wieselseweg 1107345 CC Wenum Wiesel, The Netherlands

${ }^{16}$ FitzPatrick Institute of Africa Ornithology, DST-NRF Centre of Excellence, University of Cape Town, Rondebosch 7701, South Africa

62

${ }^{17}$ MARINElife, 1 Higher Street, Bradpole, Bridport, West Dorset. DT6 3JA

64

${ }^{18}$ British Antarctic Survey, Natural Environment Research Council, High Cross, Madingley

Road, Cambridge, CB3 0ET, UK

${ }^{19}$ Bioecology and Conservation of Neotropical Birds Lab. - ICBS/UFAL - Av. Lourival Melo Mota, s/n, Tabuleiro dos Martins, CEP: 57072-900, Maceió, AL, Brazil.

${ }^{20}$ Department of Zoology, University of Oxford, New Radcliffe House, Radcliffe

Observatory Quarter, Woodstock Road, Oxford OX2 6GG, UK

${ }^{21}$ Parque Natural do Fogo- Direção Nacional do Ambiente, Chã das Caldeiras, Ilha do Fogo, Cabo Verde

${ }^{22}$ Canadian Wildlife Service, Environment and Climate Change Canada, Dartmouth, NS, B2Y 2N6, Canada

$80 \quad{ }^{23}$ Institut de Recerca de la Biodiversitat (IRBio) and Department de Biologia Evolutiva, Ecologia i Ciències Ambientals (BEECA), Universitat de Barcelona, 08028 Barcelona, Spain

${ }^{24}$ CESAM, Faculdade de Ciências, Universidade de Lisboa, 1749-016 Lisboa, Portugal

${ }^{25}$ Faculty of Biological Sciences, University of Leeds, Irene Manton Building, Leeds LS2 9JT, UK

${ }^{26}$ Wildlife Research Division, Science and Technology Branch, Environment and Climate Change Canada, Mount Pearl, NL, A1N 4T3, Canada

${ }^{27}$ Projeto Biodiversidade, Santa María, Ilha do Sal, Cabo Verde

92

${ }^{28}$ Antarctic Ecosystem Research Division, Southwest Fisheries Science Center, National

${ }^{29}$ National Institute of Polar Research, Tachikawa, Tokyo 190-8518, Japan.

${ }^{30}$ Conservation and Fisheries Department, Ascension Island Government, Ascension Island, South Atlantic ASCN 1ZZ 
${ }^{31}$ BirdLife Malta, 57/28 Triq Abate Rigord, Ta’Xbiex XBX 1120, Malta

102

${ }^{32}$ Bird Studies Canada, 17 Waterfowl Lane, Sackville, NB, E4L 1G6, Canada

104

${ }^{33}$ Antarctic Research Trust, Am Oste-Hamme-Kanal 10, 27432 Bremervörde, Germany

106

${ }^{34}$ Instituto de Biología de Organismos Marinos (IBIOMAR), CONICET, Boulevard Brown

2915, U9120ACD Puerto Madryn, Chubut, Argentina

${ }^{35}$ Department de Biologia Evolutiva, Ecologia i Ciències Ambientals (BEECA), Universitat de Barcelona, 08028 Barcelona, Spain

${ }^{36}$ University of Glasgow, Institute of Biodiversity, Animal Health and Comparative Medicine, Graham Kerr Building, Glasgow, G12 8QQ, UK.

${ }^{37}$ Centre for Ecology \& Hydrology Edinburgh, Bush Estate, Penicuik, Midlothian EH26 0QB, UK

$118{ }^{38}$ Department of Applied Biosciences, Faculty of Life Sciences, Tokyo University, 1-1-1 Izumino, Itakura-machi, Ora-gun, Gunma 374-0193, Japan 3QZ, email: steffen.oppel@rspb.org.uk 


\section{Spatial scales of marine conservation management for breeding seabirds}

\section{Introduction}

Decision makers often have to select among a suite of management actions that might benefit

a given species, and management options can range from small-scale solutions that protect a local area from outside disturbance or destruction, to large-scale or global actions that regulate human activities which are considered detrimental. In the marine realm, the unambiguous delineation of important areas for the protection of biodiversity is complicated by the lack of obvious habitat boundaries [1-3], and in many cases the spatial scale of marine protected areas is inadequate to fully protect the species of interest $[4,5]$. Selecting the most appropriate conservation management option will therefore benefit from accurate knowledge about the spatial scale at which management is required to protect highly mobile species [6].

Seabirds are distributed across all of the world's oceans and adjacent coastlines and islands $[7,8]$. They face multiple threats on land and at sea, and are more threatened than other groups of birds [9-11]. Because many seabirds feed on fish and are near the apex of the marine food chain, they are useful indicator species for the health of the marine environment and for marine spatial planning [12-14]. To protect seabirds at sea it is essential to understand their spatial distribution and potential exposure to anthropogenic threats. During the breeding season, seabirds are constrained to marine areas which they can reach from their nest while maintaining parental duties of incubating eggs or feeding chicks. The areas exploited during the breeding season are therefore important for the persistence of populations, and may be more feasible to manage than areas used during other life stages. However, some seabird species can travel thousands of kilometres even during the breeding season [e.g. 8, 15, 16], and the spatial scale of appropriate management may therefore vary.

Currently available approaches for seabird conservation at sea can be implemented across a range of spatial scales and within a variety of regulatory frameworks $[5,17]$. Areabased management approaches such as marine protected areas can be based on a broad variety of management frameworks that range from complete protection from all extractive and destructive activities ('marine reserves') to multiple use areas that permit and regulate economic activity [18-20]. For seabirds, area-based measures range from the protection of 
breeding colonies at the very local scale, to marine foraging areas around colonies and further offshore where significant seabird concentrations occur [21-23]. At larger spatial scales, additional conservation management options exist for seabirds that are not based on the protection of a specific area [17]. For example, regulations that reduce or eliminate the incidental mortality (bycatch) of seabirds in industrial or artisanal fishing operations [11, 24, 25], or regulations that limit the extraction of food resources [26, 27], can be implemented across all spatial scales and may therefore mitigate key threats to widely dispersed species [28-30]. Deciding which of these policy instruments may be most appropriate for a given seabird species of conservation concern can be informed by a better understanding of the species’ broad spatial distribution and aggregation patterns.

The distribution of seabird species was often inferred from observations at sea, until the development of small tracking devices in recent decades [31-33]. By 2017, more than 100 of the 360 species of seabirds had been equipped with tracking devices [34]; hence, sufficient seabird tracking data exist on the spatial scales of foraging to inform effective management at a broad taxonomic level $[6,35,36]$. To synthesize the existing information for management planning, two complementary aspects of seabird distribution patterns are particularly important, albeit not entirely independent: (1) the distance a species travels and the size of the marine area that birds of a given colony exploit; and (2) to what extent individuals of the same colony use the same areas at sea, which is referred to as 'spatial aggregation'. Even very mobile species can show high spatial aggregation at sea, and areas in which they congregate may be in national or international waters depending on the distance the birds travel from the colony [37, 38]. Here, seabird space-use with respect to these two aspects is quantified to indicate appropriate spatial scales for conservation management of breeding seabirds at the family level.

Existing tracking data from 52 species of ten different families collected in the Atlantic Ocean basin over the past two decades were used. These data were analysed with previously established methods $[1,39,40]$ to quantify the broad space-use requirements and spatial aggregation patterns of adult seabirds during the breeding season, and variation among families was tested. This approach allowed an assessment of whether the patterns of taxonomically coherent groups of species are sufficiently consistent to provide guidance for marine management. 


\subsection{Data collation and aggregation}

Seabird tracking data were collated for adult birds during the breeding season, when individuals are most constrained in their space-use due to the need to return to the nesting site on land. This constraint to return to the nest will likely accentuate differences between families and therefore facilitate a greater contrast in the space-use across the taxonomic spectrum. While protecting juvenile, immature and adult life-stages outside the breeding season is equally important for the conservation of long-lived species [41, 42], the movement patterns of seabirds when they are not breeding may be more affected by their latitudinal distributions than by taxonomic differences $[43,44]$. In addition, a broad taxonomic comparison of distribution patterns of juvenile, immature, and adult life stages outside the breeding season is currently difficult due to the paucity of suitably high-resolution tracking data for these stages.

Seabird tracking data from the Atlantic Ocean basin were available from the BirdLife Seabird Tracking Database [34] or through institutional repositories or collaborators. The selection of data used for this analysis was opportunistic and taxonomically imbalanced because seabird tracking efforts have so far focused on species and families of larger body size. However, the data represent a broad taxonomic spectrum of seabird movements during the breeding season from a large geographical region and are therefore useful to inform spatial scales for management.

Only tracking data from Global Positioning System (GPS) loggers and Platform Terminal Transmitters (PTT) were used due to their high spatial accuracy, and only datasets with at least five individuals were included to minimise erroneous conclusions based on small sample size [1, 37, 40]. During the breeding season, adult seabirds can be constrained to forage within different distances from their nest depending on whether they are incubating eggs or feeding small or large chicks [16, 45-47]. All tracking data were therefore divided into two stages, distinguishing the incubation period from the chick-rearing period when adults regularly return to feed the chick and therefore may not travel as far. Tracking data were analysed separately for each combination of species, colony, and breeding stage, except for some species where the tracking period spanned separate breeding stages that were not distinguished because of a lack of concurrent monitoring (classified as 'unspecified 
breeding', Table S1). Our analysis was based on 210 datasets of 52 species from ten seabird families (Tables 1 and S1).

\subsection{Rationale for space-use quantification}

The analysis to support the selection of appropriate spatial scales for conservation

102

104

106 management was designed to quantify seabird space-use in terms of (1) the distance that birds travel from their colony and the extent of the overall area that was exploited, and (2) the spatial aggregation at sea and the size of areas where a significant proportion of the population concentrated.

Although tracking data were collated from a 20-year time period, and it is possible that seabirds may have shifted their distribution in response to environmental changes over that time period [48, 49], the coarse metrics of space-use, which are based on evolutionary differences among families, were unlikely to have changed over two decades. Hence, the year in which data were collected was not considered in the analysis, and the analysis was based on the assumption that travel capabilities of the ten seabird families have not fundamentally changed between 1998 and 2017.

$<<<<$ TABLE 1 here $>>>>$

\section{Quantifying the travel distance and size of exploited area}

First, unrealistic locations were removed based on a species-specific speed filter [50] and PTT data were linearly interpolated to a regular $1 \mathrm{hr}$ interval to reduce differences between GPS and PTT data due to their different temporal sampling resolution [51]. Mean sampling schemes were one location every $17 \pm 32$ minutes (standard deviation, range 0.5 - 156) for GPS and one location every $65 \pm 34$ minutes $(2.4-188)$ for PTT datasets. Tracking data were then divided into discrete foraging trips either manually or using species- and devicespecific cut-off values for minimum distances and durations implemented with standard processing routines [1]. For each foraging trip the maximum distance from the colony (foraging range) and the total travel distance as the sum of all straight-line distances between 
all subsequent locations were calculated. The median (and range) of these trip characteristics are presented for each species, based on all foraging trips from all colonies and breeding stages, to provide a general overview of travel capabilities across seabird species [52-54]. These summaries were also calculated using just the first trip of any given individual to reduce pseudo-replication [55, 56], but this data reduction did not alter the broad taxonomic pattern (Table S2).

Because single-dimension trip characteristics do not capture the range of directionality across foraging trips from individuals in a colony, the area used by each species at each colony was also quantified. This area was calculated as the minimum convex polygon of $95 \%$ of all locations for each tracking dataset and is hereafter referred to as 'exploited area'. A minimum convex polygon was chosen to encompass less frequently used areas, and 95\% of locations were selected to avoid identifying an excessively large area due to some erratic trips or low-quality location estimates; this approach is deemed appropriate for similarly large-scale taxonomic comparisons $[57,58]$.

\subsubsection{Quantifying the spatial aggregation and size of area with concentrated use}

142 Foraging areas may range from widely dispersed to highly concentrated in a relatively restricted area. First, the ‘core area' for each individual was calculated as the 50\% kernel utilization distribution, and the extent of overlap between core areas of all individuals of a given species at a colony in a given breeding stage was then quantified. To identify the core area, the scale of the area-restricted search derived from first-passage time analysis was used as the smoothing factor in the kernel density estimator $[1,59,60]$. Because the core area size is dependent on the smoothing factor, and area-restricted search may be difficult to detect for some species or data resolutions [61], an alternative approach was also used in which the smoothing factor was scaled to the median foraging range of a colony. Results from both approaches were highly correlated and did not affect our conclusions (Table S3), and only

152 results from the former approach are presented. The overlap in core areas of individuals was quantified using Bhattacharyya’s Affinity index (BA), a non-directional measure of homerange overlap that ranges between 0 (complete separation) and 1 (completely matching probability distributions), and is considered the most appropriate index for quantifying the similarity between utilisation distributions [39, 62]. Because the BA is calculated between pairs of individuals, the BA across all pairwise comparisons was averaged for a given dataset. 
Individuals for which $<10$ locations were available were excluded from the estimation of spatial aggregation.

To compare the size of the core areas of each population (hereafter 'area of concentrated use'), the $50 \%$ kernel utilisation distribution of each individual was delineated, and areas where the $50 \%$ kernels of at least $20 \%$ of tracked individuals of that population overlapped were identified [1].

To provide a scale of reference for the marine area requirements of seabirds, the sizes of existing marine protected areas were downloaded from the World Database on Protected Areas (www.protectedplanet.net, accessed 15 Aug 2017), and filtered to include only marine and coastal protected areas.

\subsection{Assessing representativeness of datasets with varying sample size}

Sample size can affect quantitative metrics of space-use based on tracking data [40, 51, 63].

170 Because datasets ranged from 5 to 119 individuals per colony and breeding stage, the representativeness of each dataset was quantified to characterise the distribution at the level of the colony. Following the approach of Lascelles et al. [1], each dataset was iteratively subsampled to randomly select tracking data from 3 to $n-1$ individuals, where $n$ is the number of individuals tracked in that dataset. During each iteration, the $50 \%$ kernel utilisation distribution was calculated from the randomly selected data, and the proportion of the unsampled locations that fell within the $50 \%$ isopleth was assessed. If the proportion of unsampled locations contained within the $50 \%$ isopleth of the randomly selected individuals (hereafter referred to as the 'inclusion value') was $\geq 50 \%$, then the dataset was considered representative for the colony because the un-sampled individuals were already properly represented by the sampled individuals [1]. For each simulated sample size of every dataset 30 iterations were performed and the mean inclusion value across the 30 iterations was calculated for each sample size. A non-linear least-squares regression was then fitted to inclusion values to estimate the asymptote of each dataset based on the 30 iterations for each simulated sample size.

The representativeness of each dataset is reported as the proportion of the estimated asymptote that the mean inclusion value of a dataset achieved at the highest sample size. If this representativeness was $>70 \%$, a dataset was adequate to describe the space-use of the population $[1,40]$. If the non-linear regression could not identify an asymptote due to a 
singular gradient (i.e. the area expansion had not levelled off with increasing sample size), the mean inclusion value for the largest sample size of that dataset was used. The level of representativeness was then tested for a positive correlation with the number of individuals that had been tracked by calculating the Pearson correlation coefficient. The proportion of datasets for each family where the tracking data were considered not representative for the spatial distribution of a given colony is presented (Table S4).

\subsection{Statistical analysis}

To examine whether there was evidence for variation in space-use patterns at a higher taxonomic level $[64,65]$, the effect of seabird family on maximum travel distance from the colony, spatial aggregation (BA), the size of an area of concentrated use, and the size of the exploited area was tested. Generalised linear mixed models that included colony size and the stage of the breeding cycle as fixed effects were used. These two factors affect the space-use patterns of seabirds [66-68], but the analysis did not aim to investigate the relative importance of these factors and no inference was drawn from those parameters. The sampling rate of the tracking device was also included as a fixed effect because it can affect the extent and shape of home-range areas [51]. In addition, variation at the species and colony level was accounted for by including these two variables as random intercepts to avoid pseudoreplication [69]. Because some datasets had small sample sizes, each dataset was weighted based on the level of representativeness that was attained in the sensitivity analysis to reduce the influence of small and possibly unrepresentative datasets on the overall conclusions.

The data collation of all individual foraging trips was used to test the effect of seabird family on maximum travel distance from the colony. The effect of seabird family on spatial aggregation, the size of an area of concentrated use, and the size of the exploited area was tested at a population level because the latter three measures were calculated for each unique combination of species, colony, and breeding stage.

For each of these four response variables, two models were fitted that differed only by the inclusion of seabird family as a fixed factor in one of the pair of models, while all other fixed and random factors were identical. A likelihood-ratio test was used to infer whether seabird family explained a significant amount of variation in space-use variables that was not already accounted for by other fixed or random effects [70]. All analytical steps were 
conducted in R 3.4.2 [71], and code to replicate the analyses is provided at https://github.com/steffenoppel/seabirds.

\section{Results}

Seabird tracking data from 52 species across ten families were collected between 1998 and 2017 in 210 unique combinations of species, colony, and breeding stage (Table S1). The data contained a total of 12,039 distinct foraging trips from 5419 individual birds, with a mean of 21 tracked individuals (range 5 - 119) per dataset, and included $>10 \%$ of the species in each family that breed in the Atlantic Ocean basin (Table 1).

As expected, seabird species varied enormously in foraging trip characteristics, with single foraging trips ranging from $<1 \mathrm{~km}$ to $>12,000 \mathrm{~km}$ (Table 2). There was considerable variation within species and families in the foraging range, and some of this variation was explained by the breeding stage (Fig. 1). Despite substantial variation among breeding stages, species and colonies, there was clear evidence that foraging range varied at the family level (LR-Test $\chi_{9}^{2}$ $=55.57, p<0.001$ ), with cormorants having the shortest ranges, and albatrosses the largest (Table 2, Fig. 1). This pattern remained equally strong if only a single trip per individual was used in the analysis (Table S2).

$<<<<$ FIGURE 1 here $>>>>$

Seabirds also varied markedly in the extent to which they congregated at sea. The average Bhattacharyya's Affinity index for a given dataset ranged from virtually no overlap (BA < 0.001 for four datasets; Adélie Penguin Pygoscelis adeliae, European Shag Phalacrocorax aristotelis, Common Guillemot Uria aalge, Tristan Albatross Diomedea dabbenena) to very high overlap (BA = 0.91; Magellanic Penguin Spheniscus magellanicus). Due to the high variation in overlap among species and between breeding stages, there was no significant variation among families (LR-Test $\chi_{9}^{2}=12.22, p=0.20$ ). For most families there was higher overlap during chick-rearing than during incubation (Fig. 2). Cormorants, gulls and auks had consistently high overlap in both breeding stages, while albatrosses and frigatebirds showed consistently low overlap (Fig. 2). 
Owing to variability in travel distance and aggregation, the size of the marine area exploited

252

254

256

$<<<<$ FIGURE 2 here $>>>>$

Low overlap of individual core ranges can frequently lead to unrepresentative tracking data, as the foraging behaviour of untracked individuals is poorly captured by those already tracked. There were 101 (48\%) datasets that did not meet the criteria for representativeness that would be required to designate marine important bird areas following Lascelles et al. (2016), with $100 \%$ of the frigatebird datasets $(n=3)$ and $80 \%$ of albatross datasets $(n=20)$ not representative at the population level. For gulls, penguins and gannets, $>60 \%$ of datasets were representative (Table S3). There was a positive correlation between the number of individuals tracked and representativeness $\left(r_{s}=0.332, p<0.01, n=210\right)$, and of the datasets that included $>50$ individuals only three were not representative (all from albatrosses, Fig. S1).

Accounting for the level of representativeness of each dataset, and simulating the size of an area of concentrated use across a range of sample sizes, there was a strong effect of family on the size of areas of concentrated use (LR-Test $\chi_{8}^{2}=57.91, p<0.001$ ). The largest areas of concentrated use were found in albatrosses and gannets, and the smallest in cormorants and gulls, but within each family, the size of the area of concentrated use varied by two to four orders among species and breeding stages (Fig. 3).

$<<<<$ FIGURE 3 here $>>>>$

There was a negative correlation between the level of spatial aggregation at sea and the size of the area exploited during the breeding season $\left(r_{s}=-0.285, p<0.001, n=210\right)$, resulting in a gradient of space-use that can inform the relevant scales for conservation management (Fig. 
4). Species with large ranges generally had low spatial aggregation (bottom right in Fig. 4),

282

284

286

$<<<<$ FIGURE 4 here $>>>>$

\section{Discussion}

290 Seabird species range from those that congregate at sea and can be efficiently protected within a small area, to those that disperse widely and range over areas that can encompass millions of square kilometres. For the study species in the Atlantic Ocean basin, there were consistent differences among families in both maximum foraging range and the size of areas used at sea. Within this spectrum, albatrosses, petrels, storm petrels, frigatebirds, and tropicbirds travelled on average farther and dispersed more widely at sea during the breeding season than cormorants, penguins, auks, and gulls, although there was considerable variation within each family. Although some species may have recently shifted or expanded their foraging ranges due to climate-induced changes in the marine environment [48, 72], these shifts in spatial location are unlikely to be a result of fundamental changes to the species' travel capabilities, and our broad conclusions are therefore robust to climatic changes in the near future.

Our synthesis can be used to identify the management approaches likely to be most effective given the geographic scale over which the threats to a certain species need to be addressed. For some species, this broad-scale information at the family level may be sufficient to implement certain conservation actions without the need for further detailed data on individual movements from a given colony [6, 35, 73]. Some of the widely dispersing species use areas at sea that may be considered too large for the establishment and enforcement of strict marine reserves that ban all economic activity that negatively affect birds and other biodiversity [4, 19, 20]. However, other management approaches that reduce threats such as bycatch in fishing gear or depletion of prey resources can be implemented across very large spatial scales - either within appropriately managed protected areas that 
regulate rather than ban economic activities, or in the framework of other effective area-based management measures or sustainable-use regulations that apply to large marine regions without the designation of protected areas [74-77]. All management approaches should also consider that not only seabirds, but also their threats may disperse at sea and occur only in certain areas or at certain times. Static structures such as wind turbines or gillnets will affect seabirds only at one location, whereas oil, plastics and other pollutants disperse freely with currents and therefore need to be managed at different spatial scales [5]. Threats from fisheries will only occur where a particular fishery operates, and regulation of such fisheries is most important where fisheries and species vulnerable to interactions co-occur [30]. Hence, multiple management mechanisms addressing various threats in time and space may be required to safeguard particular species.

Our data represent some families better than others, and our results may not be fully representative of species-rich families such as gulls and terns, or storm petrels. For some families there may also be significant intra-family variation, which our data collation may not capture appropriately: penguins, for example, include both migrant and resident species, but our tracking data encompassed mostly migrant species, which have greater foraging ranges even during the breeding season [78]. Nonetheless, for families that encompass few species, such as the tropicbirds and the frigatebirds, the information provided here is likely more accurate and transferrable than for the gull family which encompasses $>40$ species in the Atlantic Ocean basin with a diverse range of body sizes and travel capabilities [79, 80]. Because high-resolution GPS tracking devices have only recently become small enough to track small seabirds [81], our data are biased towards larger-bodied species, with many storm petrels, small auks, and diving petrels not yet represented in tracking databases. Hence, while our study is a useful first step towards synthesizing seabird tracking data, there are some knowledge gaps where strategic tracking of certain families and species groups will advance our understanding of the space-use of smaller seabirds in the future.

Besides the incomplete coverage of all species within each family, there was large variation in the number of colonies from which tracking data for a given species were available. Seabird foraging ranges are known to vary within species, with respect to colony size and environmental factors such as ocean productivity and the foraging habitat available within a given radius [56, 66, 82-84]. The inclusion of tracking data from either a very small or a very large colony may therefore have misrepresented the typical space-use of particular species [85]. While such differences need to be considered for the implementation of specific 
protection measures, our broad scale analysis indicated that the differences in space-use among families were generally larger than differences within species, and our overall conclusions are unlikely to be affected by a few atypical datasets.

Our results also highlight that for some families the space-use patterns vary substantially between incubation and the chick-rearing stage (Figs 1 and 2, Table 2), which may be relevant for seasonal site protection or other dynamic area-based management measures that aim to regulate certain activities during discrete periods [86]. However, some of the apparent variation between breeding stages might be a consequence of varying data coverage and inter-specific differences. For example, among gannets and boobies our results seem to indicate that birds have extremely low spatial aggregation during incubation compared to brood-guard (Fig. 2). This pattern is potentially because the largest gannet in our dataset, the Northern Gannet (Morus bassanus), which forages in temperate and productive waters, has mostly been tracked while feeding chicks [87-89], whereas the available data during incubation were from the smaller, tropical boobies, which forage in less productive waters and may therefore disperse more widely at sea [90-92]. Nonetheless, our data clearly indicate that most seabirds have smaller foraging ranges and show greater aggregation at sea during the chick-rearing than the incubation period, which could be used to inform appropriate management approaches at different times of the breeding cycle .

The dataset and space-use metrics that was collated could also be used with various explanatory variables to understand the causes of variation and predict the likely movement scales of other species of seabirds for which no tracking data exist. Such extrapolations have been applied successfully to separate colonies within species [83], but if space-use requirements can also be predicted across species then some conservation management may proceed on that basis rather than await species-specific local tracking data [93, 94]. The generality of the patterns of space use found among families could be tested with data from additional species, regions and marine systems, or life-history stages. Nonetheless, researchers considering which seabirds to track for the purpose of improving conservation management are encouraged to first critically examine the value that the collected data will add to existing knowledge [35, 36, 95].

Our review focussed on adult birds during the breeding season to facilitate a broad taxonomic comparison. However, in long-lived seabirds, immature or adult birds not actively breeding may comprise a larger proportion of the total population, and may have 
fundamentally different space-use patterns and distributions than breeding adults [96-98].

Seabird conservation therefore requires not only the protection of breeding adults, but also of other life stages, which may not occur in the same spatial area. The broad scales of space-use that are summarised here for breeding adults will not be sufficient to evaluate all potential spatial overlaps with threats that may lead to population declines, and further tracking of highly threatened species or different life stages may be required to facilitate effective management [35].

In summary, seabirds are well-known indicators for the health of the marine environment [12-14], and may therefore constitute a useful tool for marine spatial planning. Many seabirds, especially cormorants, penguins, auks, and gulls congregate in certain areas at sea which are useful candidates for area-based management approaches such as marine protected areas. Marine protected areas can be managed in a variety of ways that may permit and regulate certain economic activities, and for marine protected areas of very large size, the complete exclusion of all economic activities may neither be practical nor desirable $[18,19$, 99]. Our results show that some families, especially albatrosses, petrels, storm petrels and highly pelagic tropical species such as frigatebirds and tropicbirds, disperse widely at sea, and require management approaches that are implemented at large scales such as bycatch regulations, compliance monitoring and other fisheries observer programmes, or large-scale spatial and temporal fishing closures.

\section{Acknowledgements}

398 This analysis was facilitated by the Seabird Tracking database hosted by BirdLife International (www.seabirdtracking.org). We thank many volunteers and the following individuals for assistance during data collection: Abdou Diouf, Nicolas Gomis, Moussa Samb (Direction of National Parks, Senegal), Stanislas Malou, Sokna Momie (University Cheikh

402 Anta Diop of Dakar, Senegal), Kenickie Andrews, Derren Fox, Nathan Fowler, Julia Sommerfeld, Pete Mayhew, Eelke Folmer, Almut Schlaich, Willem and Inge Bouten, Roland Gauvain, Phil Atkinson (BTO), Chantelle Burke, Bronwyn Harkness, Rielle Hoeg, Sofia Karabatsos, Amy-Lee Kouwenberg, Regan Maloney, Hilary Mann, Marina Montevecchi, Myriam Trottier-Paquet, Michelle Valliant, Skomer Wardens, Ben Dean, Holly Kirk, Robin Freeman, Dave Boyle, Chris Perrins, Helen Peat, Andrew Wood, Samir Martins, Pedro 
appreciate the financial and logistical support of the following institutions during data collection: Enterprise St Helena, Darwin Initiative, Department for Environment, Food, and Rural Affairs (UK), St Helena Nature Conservation Group, St Helena National Trust, Falkland Islands Government, Tristan da Cunha Government, South African Department of Environmental Affairs (South African National Antarctic Programme), National Research

414 Foundation (South Africa), University of Cape Town, Instituto Chico Mendes de Conservação da Biodiversidade (ICMBio/MMA), Ascension Island Government, MAVA

416 Foundation, Anguilla National Trust, Jost van Dyke Preservation Society, Alderney Commission for Renewable Energy, Alderney Wildlife Trust, The Seabird Group, Wildlife 418 Conservation Society, Department of Environment and Climate Change (Government of Canada), Bird Studies Canada, Netherlands AntArctic Program, Netherlands Organisation for 420 Scientific Research (NWO), Australian Antarctic Division, Microsoft Research Cambridge, UK Natural Environment Research Council, Northern Ireland Environment Agency, Scottish

422 Natural Heritage, Birds of Lundy Fund, Merton College (Oxford), Skokholm Islands Advisory Committee, Wildlife Trust for South and West Wales, Copeland Bird Observatory,

424 Landmark Trust, National Trust for Scotland, LIFE+ program of the European Union (LIFE10/MT/090), Maltese Ministry for Sustainable Development, the Environment and Climate Change, SPEA, Ministerio de Ciencia e Innovación and Fondos FEDER (CGL201342585-P). We thank BirdFair and the German Federal Ministry for the Environment, Nature

428 Conservation and Nuclear Safety, through the International Climate Initiative (GOBI IKI project), for the support to BirdLife International. Juan F. Masello received financial support from the German Research Foundation (DFG SPP 1158, MA2574/6-1). FCT - Portugal provided financial support through the strategic project UID/MAR/04292/2013 granted to MARE and the project PTDC/BIA-ANM/3743/2014 and project UID/AMB/ 50017/2013 and FEDER funds granted to CESAM, within the PT2020 Partnership Agreement and Compete 2020, as well as project PTDC/MAR-PRO/0929/2014. Leila Figueiredo and Marcio Amorim Efe received financial support by FAPEAL. The Grupo o Boticário de Proteção a Natureza 436 provided funds to the project granted to MAE (No. 1012_20141) via Fundação Universitária de Desenvolvimento de Extensão e Pesquisa (FUNDEPES). Ewan Wakefield was funded by the UK Natural Environmental Research Council (NERC) grant NE/M017990/1. We thank Kate Jennings, Alex Kinninmoth, Gareth Cunningham, Mike Brooke, and anonymous reviewers for constructive comments on an earlier draft of the manuscript. 
1. Lascelles, B.G., et al., Applying global criteria to tracking data to define important areas for marine conservation. Diversity and Distributions, 2016. 22(4): p. 422-431.

2. Devillers, R., et al., Reinventing residual reserves in the sea: are we favouring ease of establishment over need for protection? Aquatic Conservation: Marine and Freshwater Ecosystems, 2014. 25(4): p. 480-504.

3. Roff, J.C., M.E. Taylor, and J. Laughren, Geophysical approaches to the classification, delineation and monitoring of marine habitats and their communities. Aquatic Conservation: Marine and Freshwater Ecosystems, 2003. 13(1): p. 77-90.

4. Agardy, T., G.N. di Sciara, and P. Christie, Mind the gap: Addressing the shortcomings of

35(2): p. 226-232.

5. Boersma, P.D. and J.K. Parrish, Limiting abuse: marine protected areas, a limited solution. Ecological Economics, 1999. 31(2): p. 287-304.

6. Allen, A.M. and N. Singh, Linking movement ecology with wildlife management and conservation. Frontiers in Ecology and Evolution, 2016. 3.

7. Croxall, J.P., Seabirds: feeding ecology and role in marine ecosystems. 1987: Cambridge University Press.

8. Brooke, M.d.L., Albatrosses and petrels across the world. 2004: Oxford University Press, USA.

9. $\quad$ Croxall, J.P., et al., Seabird conservation status, threats and priority actions: a global assessment. Bird Conservation International, 2012. 22(01): p. 1-34.

10. Phillips, R.A., et al., The conservation status and priorities for albatrosses and large petrels. Biological Conservation, 2016. 201: p. 169-183.

11. Lewison, R.L., et al., Global patterns of marine mammal, seabird, and sea turtle bycatch reveal taxa-specific and cumulative megafauna hotspots. Proceedings of the National Academy of Sciences, 2014. 111(14): p. 5271-5276.

12. Parsons, M., et al., Seabirds as indicators of the marine environment. ICES Journal of Marine

13. Cairns, D.K., Seabirds as indicators of marine food supplies. Biological Oceanography, 1988.

\section{2 - 14 (4): 261-271.}

14. Frederiksen, M., R.A. Mavor, and S. Wanless, Seabirds as environmental indicators: the

15. Clay, T.A., et al., Escaping the oligotrophic gyre? The year-round movements, foraging

16. Weimerskirch, H., et al., Foraging movements of great frigatebirds from Aldabra Island:

17. Yorio, P., Marine protected areas, spatial scales, and governance: implications for the

18. Horta e Costa, B., et al., A regulation-based classification system for Marine Protected Areas

19. (MPAs). Marine Policy, 2016. 72(Supplement C): p. 192-198.

19. Lubchenco, J., et al., Plugging a hole in the ocean: The emerging science of marine reserves.

20. Gaines, S.D., et al., Designing marine reserve networks for both conservation and fisheries Gaines, S.D., et al., Designing marine reserve networks for both conservation and fisheries
management. Proceedings of the National Academy of Sciences, 2010. 107(43): p. 1828618293.

21. Pichegru, L., et al., Marine no-take zone rapidly benefits endangered penguin. Biology Letters, 2010. 6: p. 498-501. 
23. Wilson, L.J., et al., Radio-telemetry as a tool to define protected areas for seabirds in the marine environment. Biological Conservation, 2009. 142(8): p. 1808-1817.

24. Anderson, O.R.J., et al., Global seabird bycatch in longline fisheries. Endangered Species Research, 2011. 14: p. 91-106.

25. Finkelstein, M., et al., Evaluating the potential effectiveness of compensatory mitigation strategies for marine bycatch. PLoS ONE, 2008. 3(6): p. e2480.

26. Cury, P.M., et al., Global seabird response to forage fish depletion - one-third for the birds. Science, 2011. 334(6063): p. 1703-1706.

27. Sydeman, W.J., et al., Best practices for assessing forage fish fisheries-seabird resource competition. Fisheries Research, 2017. 194: p. 209-221.

28. Croxall, J., et al., Appropriate scales and data to manage seabird-fishery interactions: Comment on Torres et al. (2013). Marine Ecology Progress Series, 2013. 493: p. 297-300.

29. Torres, L.G., et al., Scaling down the analysis of seabird-fishery interactions. Marine Ecology Progress Series, 2013. 473: p. 275-289.

30. Tuck, G.N., et al., An assessment of seabird-fishery interactions in the Atlantic Ocean. ICES Journal of Marine Science, 2011. 68(8): p. 1628-1637.

31. Weimerskirch, H., et al., Foraging strategy of wandering albatrosses through the breeding season: a study using satellite telemetry. Auk, 1993. 110: p. 325-342.

32. Ropert-Coudert, Y. and R.P. Wilson, Trends and perspectives in animal-attached remote sensing. Frontiers in Ecology and the Environment, 2005. 3(8): p. 437-444.

33. Burger, A.E. and S.A. Shaffer, Application of tracking and data-logging technology in research and conservation of seabirds. Auk, 2008. 125(2): p. 253-264.

34. BirdLife International, Tracking Ocean Wanderers - The Seabird Tracking Database. 2017. McGowan, J., et al., Integrating research using animal-borne telemetry with the needs of conservation management. Journal of Applied Ecology, 2017. 54(2): p. 423-429. information for applied ecologists. Methods in Ecology and Evolution, 2015. 6(10): p. 12191228.

38. Dias, M.P., et al., Using globally threatened pelagic birds to identify priority sites for marine conservation in the South Atlantic Ocean. Biological Conservation, 2017. 211, Part A: p. 7684.

39. Wakefield, E.D., et al., Long-term individual foraging site fidelity—why some gannets don't

change their spots. Ecology, 2015. 96(11): p. 3058-3074.

40. Soanes, L.M., et al., How many seabirds do we need to track to define home-range area?

Journal of Applied Ecology, 2013. 50(3): p. 671-679.

41. Abadi, F., C. Barbraud, and O. Gimenez, Integrated population modeling reveals the impact of climate on the survival of juvenile emperor penguins. Global Change Biology, 2017. 23(3):

p. 1353-1359.

536 42. Sanz-Aguilar, A., et al., Contrasting age-specific recruitment and survival at different spatial scales: a case study with the European storm petrel. Ecography, 2009. 32(4): p. 637-646.

538 43. Alerstam, T., A. Hedenström, and S. Åkesson, Long-distance migration: evolution and determinants. Oikos, 2003. 103(2): p. 247-260.

540 44. Newton, I. and L.C. Dale, Relationship between migration and latitude among West European birds. Journal of Animal Ecology, 1996. 65(2): p. 137-146.

542 45. Granadeiro, J.P., et al., Mysterious attendance cycles in Cory's shearwater, Calonectris diomedea: an exploration of patterns and hypotheses. Animal Behaviour, 2009. 78(6): p. 1455-1462.

46. Phillips, R.A., et al., Albatross foraging behaviour: no evidence for dual foraging, and limited support for anticipatory regulation of provisioning at South Georgia. Marine Ecology Progress Series, 2009. 391: p. 279-292.

47. Boersma, P.D. and G.A. Rebstock, Foraging distance affects reproductive success in Magellanic penguins. Marine Ecology Progress Series, 2009. 375: p. 263-275. 
550 48. Louzao, M., et al., Foraging in a changing environment: habitat shifts of an oceanic predator over the last half century. Ecography, 2013. 36(1): p. 057-067.

552 49. Hazen, E.L., et al., Predicted habitat shifts of Pacific top predators in a changing climate. Nature Climate Change, 2012. 3: p. 234.

$55450 . \quad$ Shimada, T., et al., Improving data retention and home range estimates by data-driven screening. Marine Ecology Progress Series, 2012. 457: p. 171-180.

556 51. Börger, L., et al., Effects of sampling regime on the mean and variance of home range size estimates. Journal of Animal Ecology, 2006. 75(6): p. 1393-1405.

558 52. Soanes, L.M., et al., Defining marine important bird areas: Testing the foraging radius approach. Biological Conservation, 2016. 196: p. 69-79.

560 53. Thaxter, C., et al., Seabird foraging ranges as a preliminary tool for identifying candidate Marine Protected Areas. Biological Conservation, 2012. 156: p. 53-61.

562 54. Grecian, W.J., et al., A novel projection technique to identify important at-sea areas for seabird conservation: an example using Northern gannets breeding in the North East Atlantic. Biological Conservation, 2012. 156: p. 43-52.

55. Masello, J.F., et al., How animals distribute themselves in space: variable energy landscapes. Frontiers in Zoology, 2017. 14(1): p. 33.

56. Masello, J.F., et al., Diving seabirds share foraging space and time within and among species. Ecosphere, 2010. 1.

57. Nilsen, E., S. Pedersen, and J. Linnell, Can minimum convex polygon home ranges be used to draw biologically meaningful conclusions? Ecological Research, 2008. 23(3): p. 635-639.

58. Tancell, C., et al., Comparison of methods for determining key marine areas from tracking data. Marine Biology, 2013. 160(1): p. 15-26.

59. Pinaud, D. and H. Weimerskirch, Scale-dependent habitat use in a long-ranging central place predator. Journal of Animal Ecology, 2005. 74(5): p. 852-863.

60. Dragon, A., et al., Comparative analysis of methods for inferring successful foraging areas from Argos and GPS tracking data. Marine Ecology Progress Series, 2012. 452: p. 253-267.

61. Weimerskirch, H., Are seabirds foraging for unpredictable resources? Deep Sea Research Part II, 2007. 54(3-4): p. 211-223.

62. Fieberg, J. and C.O. Kochanny, Quantifying home-range overlap: the importance of the

utilization distribution. Journal of Wildlife Management, 2005. 69(4): p. 1346-1359.

63. Lindberg, M.S. and J. Walker, Satellite telemetry in avian research and management: Sample

64. Stevens, V.M., et al., A comparative analysis of dispersal syndromes in terrestrial and semi-

65. Hays, G.C., et al., Key questions in marine megafauna movement ecology. Trends in Ecology

66. Jovani, R., et al., Colony size and foraging range in seabirds. Oikos, 2016. 125(7): p. 968-

67. Phillips, R.A., et al., Causes and consequences of individual variability and specialization in

68. Brewer, M.L. and F. Hertel, Wing morphology and flight behavior of pelecaniform seabirds. Journal of Morphology, 2007. 268(10): p. 866-877.

594 69. Bolker, B.M., et al., Generalized linear mixed models: a practical guide for ecology and evolution. Trends in Ecology \& Evolution, 2009. 24(3): p. 127-135.

596 70. Lewis, F., A. Butler, and L. Gilbert, A unified approach to model selection using the likelihood ratio test. Methods in Ecology and Evolution, 2011. 2(2): p. 155-162.

598 71. R Core Team, R: A language and environment for statistical computing. 2017, R Foundation for Statistical Computing: Vienna, Austria.

600 72. Divoky, G.J., P.M. Lukacs, and M.L. Druckenmiller, Effects of recent decreases in arctic sea ice on an ice-associated marine bird. Progress in Oceanography, 2015. 136: p. 151-161.

602 73. McGowan, J. and H. Possingham, Commentary: Linking movement ecology with wildlife management and conservation. Frontiers in Ecology and Evolution, 2016. 4: p. 1-3. 
74. Abbott, J.K. and J.E. Wilen, Regulation of fisheries bycatch with common-pool output quotas. Journal of Environmental Economics and Management, 2009. 57(2): p. 195-204.

75. Moore, J.E., et al., A review of marine mammal, sea turtle and seabird bycatch in USA fisheries and the role of policy in shaping management. Marine Policy, 2009. 33(3): p. 435451.

76. Gilman, E.L., P. Dalzell, and S. Martin, Fleet communication to abate fisheries bycatch. Marine Policy, 2006. 30(4): p. 360-366.

77. Gilman, E., D. Kobayashi, and M. Chaloupka, Reducing seabird bycatch in the Hawaii

78. Croxall, J. and L. Davis, Penguins: paradoxes and patterns. Marine Ornithology, 1999. 27(1): p. 1-12.

79. Egevang, C., et al., Tracking of Arctic terns Sterna paradisaea reveals longest animal migration. Proceedings of the National Academy of Sciences, 2010: p. -.

80. Stenhouse, I.J., C. Egevang, and R.A. Phillips, Trans-equatorial migration, staging sites and 51 .

81. Bouten, W., et al., A flexible GPS tracking system for studying bird behaviour at multiple scales. Journal of Ornithology, 2013. 154(2): p. 571-580.

\section{2}

82. Lewis, S., et al., Evidence of intra-specific competition for food in a pelagic seabird. Nature, 2001. 412(6849): p. 816-819.

83. Wakefield, E.D., et al., Breeding density, fine-scale tracking and large-scale modeling reveal the regional distribution of four seabird species. Ecological Applications, 2017. 27(7): p. 2074-2091.

84. Boersma, P.D., et al., Following the fish: penguins and productivity in the South Atlantic. Ecological Monographs, 2009. 79(1): p. 59-76.

85. Weimerskirch, H., M. Le Corre, and C.A. Bost, Foraging strategy of masked boobies from the largest colony in the world: relationship to environmental conditions and fisheries. Marine Ecology Progress Series, 2008. 362: p. 291-302.

86. Game, E.T., et al., Pelagic protected areas: the missing dimension in ocean conservation. Trends in Ecology \& Evolution, 2009. 24(7): p. 360-369.

87. Cleasby, I.R., et al., Three-dimensional tracking of a wide-ranging marine predator: flight heights and vulnerability to offshore wind farms. Journal of Applied Ecology, 2015. 52(6): p. 1474-1482.

88. Hamer, K.C., et al., Contrasting foraging strategies of gannets Morus bassanus at two North Atlantic colonies: foraging trip duration and foraging area fidelity. Marine Ecology Progress Series, 2001. 224: p. 283-290.

89. Patrick, S.C., et al., Individual differences in searching behaviour and spatial foraging consistency in a central place marine predator. Oikos, 2014. 123(1): p. 33-40.

90. Mendez, L., et al., Geographical variation in the foraging behaviour of the pantropical redfooted booby. Marine Ecology Progress Series, 2017. 568: p. 217-230.

91. Oppel, S., et al., Foraging distribution of a tropical seabird supports Ashmole's hypothesis of population regulation. Behavioral Ecology and Sociobiology, 2015. 69(6): p. 915-926.

92. Soanes, L.M., et al., Important foraging areas of seabirds from Anguilla, Caribbean: Implications for marine spatial planning. Marine Policy, 2016. 70: p. 85-92. resolving management uncertainty. Journal of Applied Ecology, 2014. 52: p. 12-20. planning. Conservation Letters, 2008. 1(4): p. 190-198.

95. Latham, A.D.M., et al., The GPS craze: six questions to address before deciding to deploy GPS technology on wildlife. New Zealand Journal of Ecology, 2015. 39(1): p. 143.

654 96. Phillips, R.A., et al., Summer distribution and migration of nonbreeding albatrosses: Individual consistencies and implications for conservation. Ecology, 2005. 86(9): p. 23862396.

97. Péron, C. and D. Grémillet, Tracking through life stages: adult, immature and juvenile autumn migration in a long-lived seabird. PLoS ONE, 2013. 8(8): p. e72713. 
98. Thiebot, J.-B., et al., Larger foraging range but similar habitat selection in non-breeding versus breeding sub-Antarctic penguins. Antarctic Science, 2011. 23(2): p. 117-126.

662

99. Hobday, A.J., et al., Dynamic ocean management: Integrating scientific and technological capacity with law, policy and management. Stanford Environmental Law Journal, 2014. 33(2): p. 125-165. 